\title{
USE OF PROTON MAGNETIC RESONANCE SPECTROSCOPIC IMAGING DATA IN PLANNING FOCAL RADIATION THERAPIES FOR BRAIN TUMORS
}

\author{
EDWARD E GRAVES ${ }^{1}$, ANDREA PIRZKALL ${ }^{2}$, TRACY R MCKNIGHT ${ }^{1}$, DANIEL B VIGNERON ${ }^{1}$, \\ DAVID A LARSON ${ }^{2}$, LYNN J VERHEY ${ }^{2}$, MICHAEL MCDERMOTT ${ }^{3}$, SUSAN CHANG $^{3}$ AND SARAH J \\ NELSON $^{1}$ \\ Departments of ${ }^{1}$ Radiology, ${ }^{2}$ Radiation Oncology and ${ }^{3}$ Neurological Surgery, University of California, San \\ Francisco, CA 94143, USA \\ e-mail: nelson@mrsc.ucsf.edu \\ (Accepted May 8, 2002)
}

\begin{abstract}
Advances in radiation therapy for malignant neoplasms have produced techniques such as Gamma Knife radiosurgery, capable of delivering an ablative dose to a specific, irregular volume of tissue. However, efficient use of these techniques requires the identification of a target volume that will produce the best therapeutic response while sparing surrounding normal brain tissue. Accomplishing this task using conventional computed tomography (CT) and contrast-enhanced magnetic resonance imaging (MRI) techniques has proven difficult because of the difficulties in identifying the effective tumor margin. Magnetic resonance spectroscopic imaging (MRSI) has been shown to offer a clinically-feasible metabolic assessment of the presence and extent of neoplasm that can complement conventional anatomic imaging. This paper reviews current Gamma Knife protocols and MRSI acquisition, reconstruction, and interpretation techniques, and discusses the motivation for including magnetic resonance spectroscopy findings while planning focal radiation therapies. A treatment selection and planning strategy incorporating MRSI is then proposed, which can be used in the future to assess the efficacy of spectroscopy-based therapy planning.
\end{abstract}

Keywords: magnetic resonance imaging, magnetic resonance spectroscopy, radiation therapy.

\section{INTRODUCTION}

Neoplasms of the brain comprise 2 to $5 \%$ of all neoplastic lesions in adults and account for 11,000 deaths annually (Nelson et al., 1993). Physicians face a significant challenge in attempting to prescribe a treatment protocol that will control a central nervous system tumor and improve patient survival while maintaining the patient's nervous function and avoiding treatment-associated morbidity. The advent of three-dimensional magnetic resonance imaging (MRI) techniques for the diagnosis and followup of brain tumors has improved the clinical management of the disease. The capability of MRI to acquire images in arbitrary orientations and to vary between $\mathrm{T} 1, \mathrm{~T} 2$, and proton density contrast has greatly refined the identification of regions of neoplasm, and has aided the planning of therapies as well as post treatment followup. Furthermore, the use of intravenous magnetic resonance contrast agents has allowed more accurate differentiation of neoplasm from edematous regions.
However, identification of the presence and extent of a tumor with these techniques is subject to several pitfalls. Because other processes besides neoplasm result in degradation of the blood-brainbarrier that presents as contrast enhancement, not all visible contrast enhancement may correspond to active tumor. Both glial inflammation and radiationinduced necrosis are known to cause degeneration of the blood-brain-barrier, leading to a radiologic appearance similar to that of neoplasm. In addition, an intact blood-brain-barrier does not always indicate the absence of neoplasm. Low-grade brain tumors commonly do not exhibit contrast-enhancement, and furthermore the heterogeneity of brain tumors suggests that some regions of a neoplasm may enhance while other regions of the same lesion may not. Therefore, treatments that are directed using contrast-enhanced T1-weighted MRI may not be targeting the full extent or most active regions of the disease. Similar ambiguities are even more apparent with T2-weighted imaging. 
Magnetic resonance spectroscopy has been proposed as a complement to conventional imaging of brain tumors in order to improve the sensitivity and specificity of the diagnostic process. This technique operates by exciting and recording signals from a range of magnetic resonance frequencies, allowing the identification and quantification of several biologically significant molecules containing proton groups that resonate in the appropriate frequency range. While initial in vivo applications of spectroscopy were limited to the acquisition of a single spectrum from one volume of tissue, recent developments have combined imaging and spectroscopy acquisition techniques to allow the simultaneous recording of spectra from a threedimensional array of voxels. Magnetic resonance spectroscopic imaging (MRSI) facilitates observation of the spatial distribution of metabolic patterns over a region of interest and surrounding tissue at a high spatial resolution (1.0 $\mathrm{cc}$ nominally). A number of spectroscopic studies of brain have reported the elevation of choline and reduction of $\mathrm{N}$-acetyl aspartate (NAA) signals in neoplasm relative to normal cerebral tissue (Demaerel et al., 1991; Fulham et al., 1992; Negendank, 1992; Kinoshita et al., 1994; Vigneron et al., 1997), and have suggested use of the technique as a metabolic imaging complement to MRI.

The use of MRSI in radiation therapy presents a clinically significant and technically challenging application of this technique. Radiation is commonly used as an adjunct or stand-alone treatment for neoplasms of the brain. However, the sensitivity and specificity of the imaging technique or techniques used to identify a target to treat with radiation limit the effectiveness of focal radiotherapy techniques, such as radiosurgery. Since it has already been shown to offer metabolic information unavailable from conventional MRI, MRSI offers an alternative targetselection method and may be able to refine and improve target selection for radiation therapy. This paper first briefly reviews conventional strategies for planning radiosurgical treatments based on MRI, as well as the fundamental aspects of the acquisition and reconstruction of MRSI data. We then outline a procedure for correlating MRSI findings with treatment planning imaging datasets, which will allow designation of a radiation target volume based on spectroscopic results. The differences in target delineation using imaging- and spectroscopy-based methodologies are then discussed, and the implications of these differences for clinical applications such as radiosurgery are examined. Finally, a novel treatment strategy incorporating MRSI for both the selection of therapy and for the planning of radiosurgery is proposed.

\section{GAMMA KNIFE RADIOSURGERY PLANNING AND DELIVERY}

At UCSF, the clinically-utilized radiosurgical unit is the Gamma Knife (Elekta Instruments, Atlanta, GA). This device allows the delivery of a highly conformal dose distribution to an irregular target with minimal dose to the surrounding tissue. For glioma patients, Gamma Knife (GK) treatment is typically administered as a stand-alone treatment at the time of tumor recurrence or as a boost during post-resection radiotherapy. The Radiation Therapy Oncology Group (RTOG) has defined criteria in order to select patients for Gamma Knife treatment who will have the best response to the therapy. These include 1) patient Karnofsky Performance Status (KPS) greater than 60,2 ) a well-circumscribed unilateral tumor less than $4 \mathrm{~cm}$ in its greatest diameter with no subependymal spread or leptomeningeal extension, and 3) a location not adjacent to the optic chiasm or brain stem (Larson et al., 1996).

The morning of treatment, a stereotactic frame is attached to the patient's skull to provide a means of immobilization as well as a coordinate reference system. The patient then undergoes an MR examination, which is subsequently transferred to a workstation for treatment planning using the GammaPlan software package. Treatment planning is accomplished using primarily the contrast-enhanced T1-weighted MR, outlining the contrast enhancement plus any additional area of concern according to the judgement of the radiation oncologist and/or neurosurgeon. After specification of a desired target, a sequence of individual radiation "shots" is planned such that the resultant dose distribution (typically the 50\% isodose line) conforms to the target volume. The prescription dose is then specified in inverse relation to the size of the target. As an example, Fig. 1 displays an axial slice of the dose distribution prescribed to the contrast enhancement in a patient with recurrent glioma. Following construction of a treatment plan, the patient is positioned within the Gamma Knife using the head frame, and the shots required to generate the desired dose distribution are delivered sequentially.

\section{ACQUISITION OF MRSI DATA}

Chemical shift imaging (CSI) or magnetic resonance spectroscopic imaging (MRSI) has been developed over the last fifteen years, and is currently being released commercially on a number of scanner platforms. 


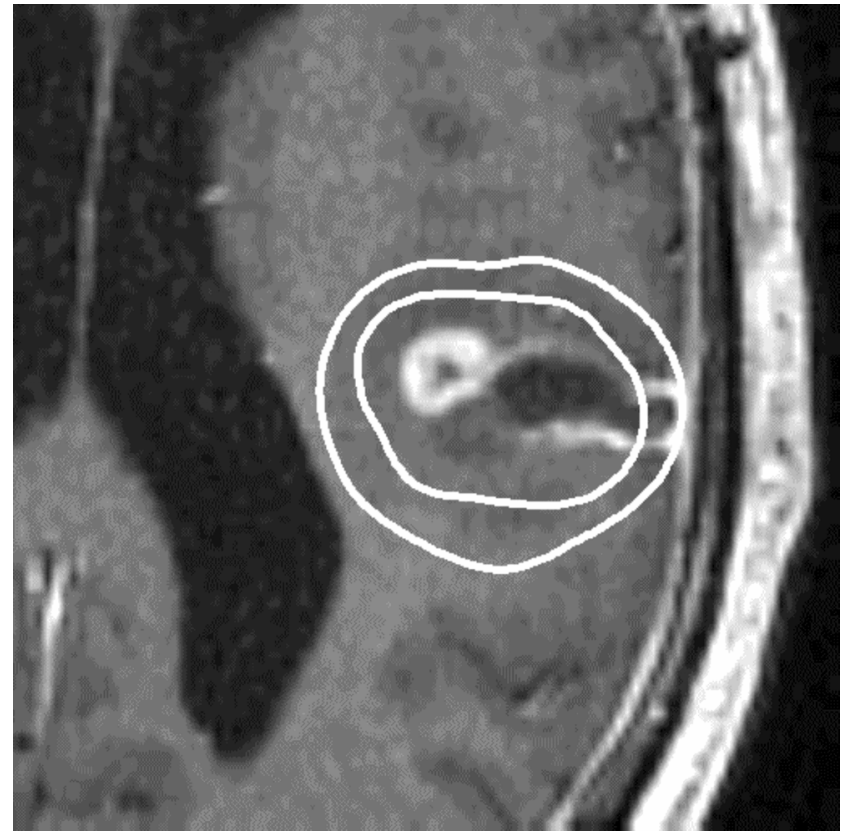

Fig. 1. A Gamma Knife dose distribution delivered to a patient with a grade III glioma. This treatment plan was prescribed based on the contrast-enhanced T1weighted MRI, which is shown. The inner and outer contours depict the $50 \%$ and $25 \%$ isodose lines. The maximum dose delivered to this patient was $35 \mathrm{~Gy}$.

Recent technological advances have improved the field-of-view, acquisition time, and data quality of MRSI to levels where it can be used for clinical assessment of brain tumors. At our institution, a three-dimensional MRSI acquisition employing a point-resolved spectroscopy (PRESS) technique for volume localization (Bottomley, 1987) has been developed on a Signa Echospeed 1.5T clinical MR scanner (General Electric Medical Systems, Milwaukee, WI). Spectral- and spatial-selective $180^{\circ}$ pulses are used to excite the frequency range of metabolic interest while leaving the water resonance at $4.7 \mathrm{ppm}$ unexcited (Star-Lack et al., 1997). Trains of very-selective saturation (VSS) pulses (Tran et al., 2000) are applied before the $90^{\circ}$ excitation pulse in spatial bands surrounding the PRESS-selected region for outer volume suppression. To conform the shape of the excited region so that maximum coverage of the region-of-interest can be obtained while avoiding subcutaneous fat or other spectrally undesirable regions, graphically prescribed VSS bands are also used.

The PRESS-selected region is chosen based on MR images acquired during the same examination to include as much of the region of interest as possible as well as contralateral and other tissue removed from the morphologic abnormality, while avoiding bone, subcutaneous fat, or other materials that would complicate shimming and water suppression. A phase encoding matrix of $8 \times 8 \times 8,16 \times 8 \times 8$, or $12 \times 12 \times 8$ is chosen based on the dimensions of the volume to be excited. The excitation volume can be at maximum $80 \%$ of the size of the acquisition matrix in order to avoid frequency aliasing (Noworolski, 1999). The use of a rectangular $\mathrm{k}$-space sampling trajectory prevents the acquisition of larger phase-encoding matrices because of time constraints. Accurate placement of the PRESS box is verified on the imaging sequence acquired just prior to the MRSI sequence. Automated shimming is performed over the region to be excited just prior to the spectral acquisition. All MRSI acquisitions use a TR of $1000 \mathrm{~ms}$, a TE of $144 \mathrm{~ms}$, and are acquired in 17-19 min. The nominal spatial resolution of MRSI data acquired with the head coil is $1.0 \mathrm{cc}$. The spectral width used is $1000 \mathrm{~Hz}$, and 512 points are acquired per spectrum. The additional time required in order to perform MRSI during a clinical MR imaging examination is approximately $30 \mathrm{~min}$., resulting in total MRI/MRSI examination times of 1.0 $-1.5 \mathrm{hrs}$.

\section{RECONSTRUCTION OF MRSI DATA}

Currently, reconstruction and analysis of threedimensional MRSI data is achieved by transferring the data from the scanner to an offline UltraSPARC Solaris workstation (Sun Microsystems, Palo Alto, CA) and using software developed for research purposes. However, efforts are underway to implement these analysis procedures on the acquisition platform. Initially, all MRI data acquired in the examination are automatically aligned (Nelson et al., 1994) to the image acquired just before the MRSI data. The reference image for this process is chosen since it is assumed to be the most similar in spatial orientation to the spectral data. This procedure corrects for any patient motion between acquisitions, and effectively brings all data in the coordinate frame of the MRSI data. Reconstruction of the MRSI data includes zerofilling of each sampled FID to 1024 points and apodization in the time domain using a $2 \mathrm{~Hz}$ Lorentzian filter, followed by Fourier transformation along each of the three k-space dimensions and the time dimension. The resulting frequency spectra are then automatically frequency, phase, and baseline corrected on a voxel-by-voxel basis. The peak parameters (height, area, and width) for choline (Cho), creatine (Cr), $\mathrm{N}$-acetyl aspartate (NAA), and lactate/lipid (LL) are then calculated for each voxel in the final, corrected MRSI dataset.

Efforts have been made to design an analysis technique that can assign a quantitative measure of abnormality to a spectrum, facilitating simple and 
quick assessment of the metabolic lesion from a complex, four-dimensional MRSI dataset. Towards this end, a statistical routine has been developed that computes a measure of abnormality for each spectral voxel in a dataset based on the extent to which its choline and NAA levels, quantified in terms of peak area, deviate from those of an automatically selected control normal population of voxels. This measure is referred to as the choline/NAA residual, and has been shown to reproducibly quantify the degree of spectral abnormality in a voxel relative to normal voxels identified in the same dataset (McKnight et al., 2001). The metric is reported as the number of standard deviations a voxel differs from the control voxels. At present, a cutoff of 2.0 is used to separate voxels consistent the presence of neoplasm from those consistent with necrosis or healthy tissue.

\section{CORRELATION OF MRSI WITH TREATMENT PLANNING MRI}

In order to apply MRSI towards radiation treatment planning, a method for viewing this complex data in the framework of image-based treatment planning software packages must be in place. We have accomplished this through a simple and generally applicable technique (Graves et al., 2001). Following registration of the MRI component of the MRI/MRSI examination to the MRI dataset being used for GK treatment planning, the spectral dataset is registered using the same transformation. The choline/NAA residual map determined from the registered MRSI data is then resampled to the resolution of the registered MRI, and represented as a contour map at levels of 2.0, 3.0, and 4.0. The contours are represented within the registered MRI pixel data, and the final image is sent to the treatment planning workstation. There it can be viewed in the same coordinate frame as the planning image dataset, and the spectral contours can be used to guide the delineation of a radiation target. This technique was been shown to reproducibly identify the region of spectroscopic abnormality in glioma patients to within $3 \mathrm{~mm}$, allowing irradiation of this region by targeting of the relevant spectral contour plus a small margin (Graves et al., 2001). Since the technique relies solely on images to convey the metabolic information, it is applicable to any treatment planning system capable of viewing multiple imaging datasets.

\section{DIFFERENCES BETWEEN ANATOMIC AND METABOLIC LESIONS IN BRAIN TUMORS}

Our group has acquired more than 1500 combined MRI/MRSI examinations of patients with brain tumors, and has found that there are often substantial differences between the spatial extent of metabolic and anatomic lesions. It has been reported that of 100 patients with recurrent primary brain tumors, $36 \%$ of patients with high-grade lesions and $56 \%$ of patients with mid or low grade lesions had spectra with elevated choline and decreased NAA outside the region of contrast-enhancement (Nelson et al., 1999). A separate analysis using the choline/ NAA residual metric reported that although there were focal areas with high residuals both inside and outside the volume of contrast enhancement, it was common that only about $50 \%$ of the T2 hyperintense region had voxels with a choline/NAA residual greater than or equal to 2.0 (McKnight et al., 2001). This suggests that MRSI may be useful for both expanding high dose treatment volumes beyond the contrast-enhancing region and limiting the dose delivered to cover only a subset of the $\mathrm{T} 2$ hyperintense volume. These findings are to be expected based upon the underlying biological processes, and provide an estimate of how frequently the region of contrast enhancement is likely to be inadequate as a target for radiosurgery.

These concepts were further explored in a study comparing the contrast-enhancing and T2-hyperintense regions with regions with choline/NAA residual values greater than 2.0, 3.0 and 4.0 in a population of patients with newly diagnosed grade III and grade IV gliomas (Pirzkall et al., 2001). This analysis showed that while the choline/NAA residual 2.0 contour was in general much smaller than the T2-hyperintense volume, there were also regions with a choline/NAA residual of 2.0 outside of the T2 volume. For grade III lesions, the metabolic lesions were much larger than the contrast-enhancing regions and extended beyond it by as much as $30 \mathrm{~mm}$. For grade IV lesions, there was less overlap between the contrast-enhancing and spectroscopically abnormal regions. This is presumably due to the prevalence of micronecrosis in these tumors, leading to lower metabolite levels within the volume of contrast enhancement. In these cases, the contrast-enhancing region was relatively larger than for the patients with grade III tumors, and 
the spectroscopic abnormalities did not extend as far beyond the enhancement $(18 \mathrm{~mm}, 14 \mathrm{~mm}$ and $10 \mathrm{~mm}$ for residuals of 2.0, 3.0, and 4.0, respectively).

\section{IMPLICATIONS OF METABOLIC TARGET DELINEATION FOR GAMMA KNIFE RADIOSURGERY}

These findings suggest that integration of MRSI data into the radiation treatment planning process may be critical for accurately defining the radiation target. We have previously investigated the prognostic significance of the observation of abnormal spectra beyond the radiosurgical target (Graves et al., 2000). The pre-treatment metabolic lesions of 36 glioma patients undergoing Gamma Knife radiosurgery were compared to the radiation target volume, and to the patients' clinical outcome. The results of this analysis demonstrated a tendency for poorer responses, in terms of survival time, time-to-further-treatment, and change in contrast-enhancing volume, among patients with spectroscopic abnormalities that extended beyond the target volume at the time of treatment. An example of the poor outcomes observed following radiosurgery for patients with extensive metabolic abnormalities is shown in Fig. 2. This data gives justification for selecting and planning treatments based on spectroscopic findings.

Fig. 2. Contrast-enhanced T1-weighted imaging and MRSI results for a grade III glioma patient before and after Gamma Knife treatment. The 15 Gy isodose line is shown in white. Voxels with spectra with choline/NAA residuals greater than 2.0 are marked with an asterisk. Following treatment, both MRI and MRSI display a significant worsening in the patient's status. Planning radiosurgery to the metabolic rather than the anatomic lesion may improve patient response to therapy.
Because Gamma Knife radiosurgery is a focal ablative therapy, modification of the prescribed radiation target based on MRSI data has proceeded cautiously. It is important to note that because only targets of diameter less than or equal to $4 \mathrm{~cm}$ can be effectively treated with radiosurgery, a patient's anatomic lesion may meet this criterion while the spectroscopic lesion may exceed this limiting size. This is demonstrated in Fig. 3, which depicts two cases in which consideration of the spectroscopic abnormality expands the effective target. However, in case $\mathrm{A}$ it remains feasible to treat the enlarged target with radiosurgery, while in case B this is no longer an option. To date, MRSI-based modification of a Gamma Knife treatment plan has in general proceeded from an already-prescribed treatment plan based on contrast-enhanced T1-weighted MRI findings. The constructed plan is then viewed superimposed on the choline/NAA residual contour-labeled MRI image within GammaPlan, and the dose distribution is modified at the discretion of the radiation oncologist and/or neurosurgeon to include regions of spectral abnormality outside the conventional target. This is done subjectively, although it is valid to generalize that treatment planning personnel are more likely to alter a target if the spectral abnormality possessed a residual level greater than 3.0, a volume of at least $1.0 \mathrm{cc}$ outside the conventional target, and if the extension was physiologically reasonable.

Procedures for incorporating spectral information into the clinical decision-making process for Gamma Knife radiosurgery are currently being developed. A treatment selection methodology has been proposed for newly diagnosed and recurrent grade IV radiosurgery candidates that considers pre-treatment MRSI results, although only in treatment selection and not in the prescription of the radiosurgical target (McDermott et al., 2000). In this plan, patients with more extensive spectroscopic lesions would receive external beam irradiation in addition to radiosurgery to treat the metabolic abnormalities beyond the radiologic lesion. In situations where the extent of abnormal spectroscopic voxels is larger than the maximum permissible target volume for radiosurgery, the strategy suggests the use of concurrent chemotherapy or the use of chemotherapy as an alternative to radiosurgery in cases of tumor recurrence. Fig. 4 describes a treatment strategy including elements of the previously suggested plan, but which also utilizes MRSI data to modify the radiosurgical target volume in certain cases. A small number of patients have been treated using this strategy, and while preliminary, the outcomes of these patients have encouraged us to further pursue MRSI-defined Gamma Knife treatments. 
GRAVES EE ET AL: MRSI in planning radiation therapy

Fig. 3. Contrast-enhanced T1-weighted imaging and MRSI results for two patients treated with Gamma Knife radiosurgery. Voxels with spectra with choline/NAA residuals greater than 2.0 (i.e., suggestive of tumor presence) are marked with an asterisk. The contours shown in A) are the $25 \%$ and $50 \%$ isodose lines, while the contour in $B$ ) is the $25 \%$ isodose line. The extent of the abnormal voxels for the grade III glioma patient shown in A) is such that the entire spectroscopic abnormality could be treated with Gamma Knife. The grade IV glioma patient shown in B) however possesses a spectroscopic abnormality that exceeds the size restriction for this form of radiosurgery.

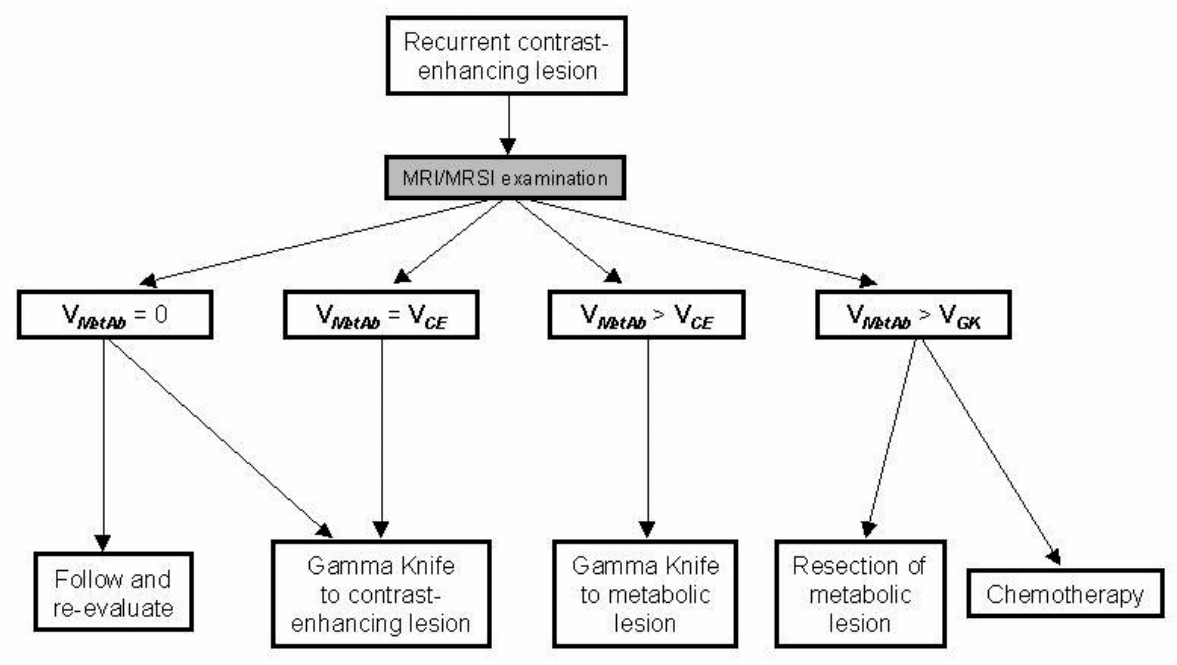

Fig. 4. A flowchart depicting a possible treatment selection and planning strategy that incorporates MRSI findings. The final nodes for each path depict possible courses of action in the treatment of brain tumor patients displaying the corresponding diagnostic features. $V_{\text {Metab }}$ : volume of the metabolic abnormality as determined by MRSI, $V_{C E}$ : volume of contrast enhancement, $V_{G K}$ : maximum permissible Gamma Knife target volume. 


\section{CONCLUSIONS}

From the results that have been summarized here, it is clear that MRSI is a clinically feasible technique that provides information of interest for planning focal radiation therapy. The spectral data differ from both the contrast enhancing and T2-hyperintense regions, both in terms of intensity and spatial extent. The differences between the anatomic and metabolic lesions suggest that incorporation of MRSI in the definition of radiation target volumes may allow more effective targeting and destruction of tumor cells while also more effectively sparing normal brain tissue. While the choline/NAA residual appears to be a useful index, the spatial distribution of the MRSI creatine resonance may also be relevant for defining radiation sensitivity, since it may reflect the degree of oxygenation within the lesion ( $\mathrm{Li}$ et al,. 2001). Although the spatial resolution and signal to noise ratio of MRSI is relatively low compared to other conventional imaging techniques, increases in main field strength and the use of surface radiofrequency coils can help overcome these limitations. An attractive feature of MRSI is that it can be performed during a clinical MR examination and can therefore be combined with other anatomic or physiologic MR imaging methods that may provide insight in the treatment planning process, such as cerebral blood volume imaging and diffusion-weighted imaging. Because of the significant clinical value of the magnetic resonance spectroscopic imaging applications in radiation therapy discussed here, as well as in other forms of targeted therapy, continued research in this field is warranted.

\section{ACKNOWLEDGEMENTS}

The authors would like to acknowledge the contributions of Evelyn Proctor, Niles Bruce, and Umberto Jiminez, as well as the support of grant RO1 CA59880 from the National Institutes of Health.

\section{REFERENCES}

Bottomley PA (1987). Spatial localization in NMR spectroscopy in vivo. Ann N Y Acad Sci 508:333-48.

Demaerel P, Johannik K, Van Hecke P, Van Ongeval C, Verellen S, Marhcal G, Wilms G, Plets C, Goffin J, Van Calenbergh F, Lammens M, Baert AL (1991). Localized 1H NMR spectroscopy in fifty cases of newly diagnosed intracranial tumors. J Comput Assist Tomog 15:67-76.

Fulham MJ, Bizzi A, Dietz MJ, Shih HH, Raman R, Sobering GS, Frank JA, Dwyer AJ, Alger JR, Di Chiro
G (1992). Mapping of brain tumor metabolites with proton MR spectroscopic imaging: clinical relevance. Radiology 185:675-86.

Graves EE, Nelson SJ, Vigneron DB, Chin C, Verhey L, McDermott M, Larson D, Sneed PK, Chang S, Prados MD, Lamborn K, Dillon WP (2000). A Preliminary Study of the Prognostic Value of Proton Magnetic Resonance Spectroscopic Imaging in Gamma Knife Radiosurgery of Recurrent Malignant Gliomas. Neurosurgery 46:319-28.

Graves EE, Pirzkall A, Nelson SJ, Larson D, Verhey L (2001). Registration of magnetic resonance spectroscopic imaging to computed tomography for radiotherapy treatment planning. Medical Physics 28:2489-96.

Kinoshita Y, Kajiwara H, Yokota A, Koga Y (1994). Proton magnetic resonance spectroscopy of brain tumors: an in vitro study. Neurosurgery 35:606-13.

Larson DA, Gutin PH, McDermott M, Lamborn K, Sneed PK, Wara WM, Flickinger JC, Kondziolka D, Lunsford LD, Hudgins WR, Friehs GM, Haselberger K, Leber K, Pendl G, Chung SS, Coffey RJ, Dinapoli R, Shaw EG, Vermeulen S, Young RF, Hirato M, Inoue HK, Ohye C, Shibazaki T (1996). Gamma knife for gliomas: Selection factors and survival. Int $\mathrm{J}$ Radiat Oncol Biol Phys 36:1045-53.

Li X, Lu Y, Nelson SJ (2001). Analysis of Spatial Extent of the Metabolic Abnormality for Newly Diagnosed Glioma Patients. International Society of Magnetic Resonance in Medicine Seventh Annual Meeting, Glasgow, Scotland, U.K.:2295.

McDermott MW, Chang SM, Keles GE, Graves EE, Nelson SJ, Larson DA, Berger MS (2000). Gamma Knife Radiosurgery for Primary Brain Tumors. In: Germano IM, ed. LINAC and Gamma Knife Radiosurgery. Park Ridge: American Association of Neurological Surgeons, 189-202.

McKnight TR, Noworolski SM, Vigneron DB, Nelson SJ (2001). An automated technique for the quantitative assessment of 3D-MRSI data from patients with glioma. J Magn Reson Imaging 13:167-77.

Negendank W (1992). Studies of human tumors by MRS: a review. NMR Biomed 5:303-24.

Nelson DF, McDonald JV, Lapham LW, Qazi R, Rubin P (1993). Central Nervous System Tumors. In: Rubin P, ed. Clinical Oncology, 7th Edition. Philadelphia: W.B. Saunders Company, 617-44.

Nelson SJ, Nalbandian AB, Proctor E, Vigneron DB (1994). Registration of images from sequential MR studies of the brain. J Magn Reson Imaging 4:877-83.

Nelson SJ, Vigneron DB, Dillon WP (1999). Assessment of Brain Tumors Using Volume MRI and MRSI. NMR Biomed 12:123-38.

Noworolski SM (1999). High Resolution Magnetic Resonance Imaging and Spectroscopy Using Surface Coils. 
Pirzkall A, McKnight TR, Graves EE, Carol MP, Sneed PK, Wara WW, Nelson SJ, Verhey LJ, Larson DA (2001). MR-spectroscopy guided target delineation for high-grade gliomas. International Journal of Radiation Oncology Biology Physics 50:915-28.

Star-Lack J, Vigneron DB, Pauly J, Kurhanewicz J, Nelson SJ (1997). Improved solvent suppression and increased spatial excitation bandwidths for three-dimensional PRESS CSI using phase-compensating spectral/spatial spin-echo pulses. J Magn Reson Imaging 7:745-57.
Tran TK, Vigneron DB, Sailasuta N, Tropp J, Le Roux P, Kurhanewicz J, Nelson S, Hurd R (2000). Very selective suppression pulses for clinical MRSI studies of brain and prostate cancer. Magn Reson Med 43:2333.

Vigneron DB, Nelson SJ, Kurhanewicz J (1997). Proton chemical shift imaging of cancer. In: Higgins CB, Hricak H, Helms CA, eds. Magnetic Resonance Imaging of the Body, Third Edition. New York: Lippincott-Raven Press, 205-20. 